\title{
A Coupled Line Impedance Transformer for High Termination Impedance with a Bandpass Filtering Response
}

\author{
Phirun Kim · Yongchae Jeong*
}

\begin{abstract}
In this study, a short-ended coupled line with a short-circuit stub transmission line bandpass filtering impedance transformer is presented. The general designed equations are derived on the basis of circuit theory. The design curves are provided to examine the characteristic of the proposed impedance transformer. The proposed circuit is suitable for high termination impedance. To validate the design formulas, a 400-50 $\Omega$ impedance transformer is designed and fabricated at the operating center frequency $\left(f_{0}\right)$ of 2.6 GHz. The measured results show a good agreement with the simulation. The measured insertion and return losses are $0.6 \mathrm{~dB}$ and $22.5 \mathrm{~dB}$ at $f_{0}$, respectively. The measured return loss is higher than $20 \mathrm{~dB}$ within the passband frequency of 2.51-2.7 GHz. Moreover, the stopband attenuation is higher than $25 \mathrm{~dB}$ from $\mathrm{DC}$ to $1.64 \mathrm{GHz}$ of the lower stopband and from $3.12 \mathrm{GHz}$ to $6.4 \mathrm{GHz}$ of the higher stopband.
\end{abstract}

Key Words: Bandpass Response, Coupled Line, Impedance Transformer, Transmission Pole.

\section{INTRODUCTION}

The bandpass filtering impedance transformer (IT) is rapidly developing in modern communication systems. This IT is increasingly becoming necessary in many applications, such as power dividers [1], antenna feeding lines [2], and amplifier design [3]. Indeed, a conventional quarter-wavelength transformer is a well-known IT [4], but this network has some limitations, such as difficulty in the realization of a high impedance transforming ratio and poor out-of-band suppression. In recent years, various structures of filtering IT have been proposed [5-11]. A mixed lumped and distributed IT with low-pass filtering response is designed in [5]. However, the shunt capacitors can produce a self-resonance frequency, which introduces additional parasitic effects at high frequency. Wideband ITs are proposed using an open-circuit parallel-coupled line with an interconnecting transmission line (TL) [6] and a multiple-section stepped-impedance TL with input/output couplings [7]. However, their impedance transforming ratio is low. In [8], an open-circuit stub coupled-line IT with a bandpass filtering response is designed to transform $20 \Omega$ to $50 \Omega$. In [9], a bandpass response coupled-line IT with an ultra-high impedance transforming ratio is presented by using two-section open-ended coupled lines. This IT is applicable to low termination impedance. Therefore, a short-ended coupled-line IT with an ultra-high impedance transforming ratio is proposed [10] for high load termination impedance. High selectivity is obtained by increasing the number of stages, but the insertion loss is degraded. Moreover, the stopband characteristic is limited. In [11], a bandpass filtering IT is designed with a section of open-ended coupled lines and an open-circuit stub TL.

Manuscript received July 5, 2017 ; Revised September 26, 2017 ; Accepted December 24, 2017. (ID No. 20170705-029J)

Division of Electronics and Information Engineering, IT Convergence Research Center, Chonbuk National University, Jeonju, Korea.

"Corresponding Author: Yongchae Jeong (e-mail: ycjeong@jbnu.ac.kr)

This is an Open-Access article distributed under the terms of the Creative Commons Attribution Non-Commercial License (http://creativecommons.org/licenses/by-nc/4.0) which permits unrestricted non-commercial use, distribution, and reproduction in any medium, provided the original work is properly cited.

(c) Copyright The Korean Institute of Electromagnetic Engineering and Science. All Rights Reserved. 
The transformer is valid for low termination impedances at the source and/or load terminals.

In this study, an IT with a short-circuit stub parallel-coupled line and a TL with a bandpass filtering response is presented. The proposed network can be applied to high load termination impedance. A short-circuit stub TL can produce several transmission zeros in the stopband. Moreover, a bandpass filtering response with high selectivity can be obtained.

\section{DESIGN EQUATION}

Fig. 1(a) shows the schematic of the proposed IT, which is composed of a short-end parallel-coupled line and a short-circuit stub TL with a characteristic admittance of $Y_{1}$ connected at coupling port 2 of the coupled line. The electrical lengths of the parallel-coupled line $(\theta)$ and the short-circuit stub TL $(2 \theta)$ are given as a quarter-wavelength $(\lambda / 4)$ and a half-wavelength $(\lambda / 2)$ at $f_{0}$, respectively. A short-circuit stub TL is used to create two transmission poles in the passband and three transmission zeros in the stopband [11], where the transmission zeros are located on the lower and upper sides of the passband, respectively. The proposed circuit is efficient at high termination impedance. Fig. 1(b) shows the schematic of the impedance transformer in [11] with an open-end parallel-coupled line and an open-circuit stub TL. The network is used only for low termination impedance.

The reflection and transmission coefficients of the proposed IT network, where input port 1 and output port 3 are terminated with the admittances $Y_{S}$ and $Y_{L}$, respectively, can be derived as (1).

$$
\begin{aligned}
& S_{11}=\frac{\left(Y_{11}-Y_{S}\right)\left(Y_{22}+r Y_{S}\right)-Y_{12} Y_{21}}{\left(Y_{11}+Y_{S}\right)\left(Y_{22}+r Y_{S}\right)-Y_{12} Y_{21}} \\
& S_{21}=\frac{2 Y_{21} \sqrt{Y_{S} Y_{L}}}{\left(Y_{11}+Y_{S}\right)\left(Y_{22}+r Y_{S}\right)-Y_{12} Y_{21}},
\end{aligned}
$$

where

$$
\begin{gathered}
Y_{11}=j \frac{\cot \theta}{2}\left[\frac{\left(Y_{0 o}-Y_{0 e}\right)^{2} \cot \theta}{2 Y_{1} \cot 2 \theta+\left(Y_{0 e}+Y_{0 o}\right) \cot \theta}-\left(Y_{0 e}+Y_{0 o}\right)\right] \\
Y_{12}=Y_{21}=j \frac{Y_{0 o}-Y_{0 e}}{2} \csc \theta\left[\frac{\left(Y_{0 e}+Y_{0 o}\right) \cot \theta}{2 Y_{1} \cot 2 \theta+\left(Y_{0 e}+Y_{0 o}\right) \cot \theta}-1\right] \\
Y_{22}=j \frac{Y_{0 e}+Y_{0 o}}{2}\left[\frac{\left(Y_{0 e}+Y_{0 o}\right) \csc ^{2} \theta}{2 Y_{1} \cot 2 \theta+\left(Y_{0 e}+Y_{0 o}\right) \cot \theta}-\cot \theta\right] \\
\theta=\frac{\pi}{2} \frac{f}{f_{0}} .
\end{gathered}
$$

Moreover, $Y_{S}=1 / Z_{S}, Y_{L}=1 / Z_{L}, Y_{0 e}=1 / Z_{0 e}$, and $Y_{0 o}=1 / Z_{0 o}$. By solving (1a), the even-mode characteristic admittance $Y_{0 e}$ of the coupled line with the specified $S_{11}, Y_{S}$, and $r$ at $f_{0}$ can be derived

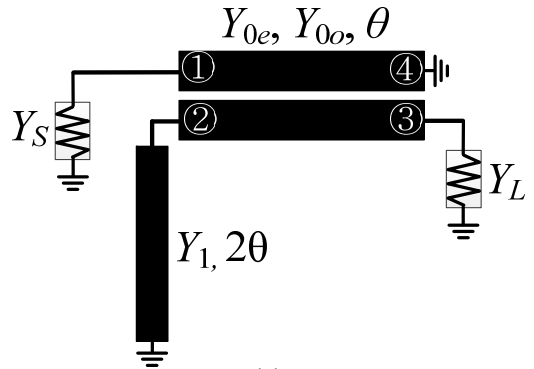

(a)

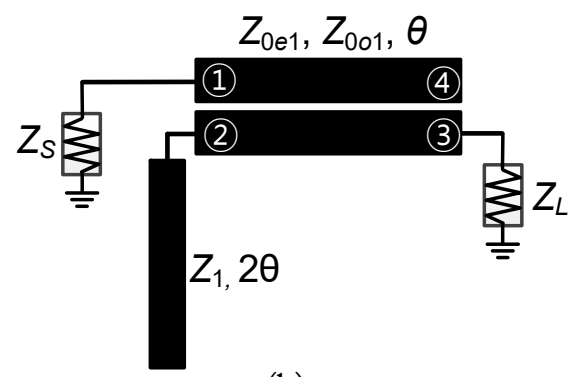

(b)

Fig. 1. Schematic of the impedance transformer. (a) Proposed network and (b) network in [11].

as (3).

$$
Y_{0 e}=Y_{0 o}-2 Y_{S} \sqrt{\frac{r\left(1+\left.S_{11}\right|_{f=f_{0}}\right)}{\left(1-\left.S_{11}\right|_{f=f_{0}}\right)}},
$$

where

$$
r=\frac{Y_{L}}{Y_{S}}=\frac{Z_{S}}{Z_{L}} .
$$

$r$ is an impedance transforming ratio of $Z_{S}$ to $Z_{L}$. $S_{11}$ and $Y_{0 o}$ are the predefined magnitude of return loss and odd-mode admittance of the coupled line at $f$, respectively. By giving (1a) equal to zero, the characteristic admittance of $Y_{1}$ can be derived as (5).

$$
Y_{1}=\frac{Y_{0 e}+Y_{0 o}}{r-1} .
$$

Using (5), two transmission poles can be obtained in the passband [11].

From the above design equations, the relationship of $Z_{0 e}$ and $Z_{0 o}$ with $r$ is plotted in Fig. 2. $Z_{0 e}$ dramatically decreases as $r$ increases. With the same $r, Z_{0 e}$ increases as $Z_{00}$ increases. Therefore, a higher $r$ requires a relatively looser coupling than a lower $r$, where the coupling coefficient is given by $C=20 \log \left[\left(Z_{0 e}-Z_{0 o}\right)\right.$ $\left./\left(Z_{0 e}+Z_{0_{0}}\right)\right][\mathrm{dB}]$. The calculation is conducted by choosing $Z_{L}$ $=50 \Omega, S_{11}=-20 \mathrm{~dB}$ at $f_{0}, Z_{0 o}=27 \Omega, 30 \Omega, 35 \Omega$, and $r$ varies from 2 to 12 .

Similarly, Fig. 3 shows the variations of $Z_{1}$ according to $r$ and $Z_{00} . Z_{1}$ increases as $r$ increases. Moreover, $Z_{1}$ increases more with a higher $Z_{00}$. As shown in Figs. 2 and 3, a low $r$ prevents 


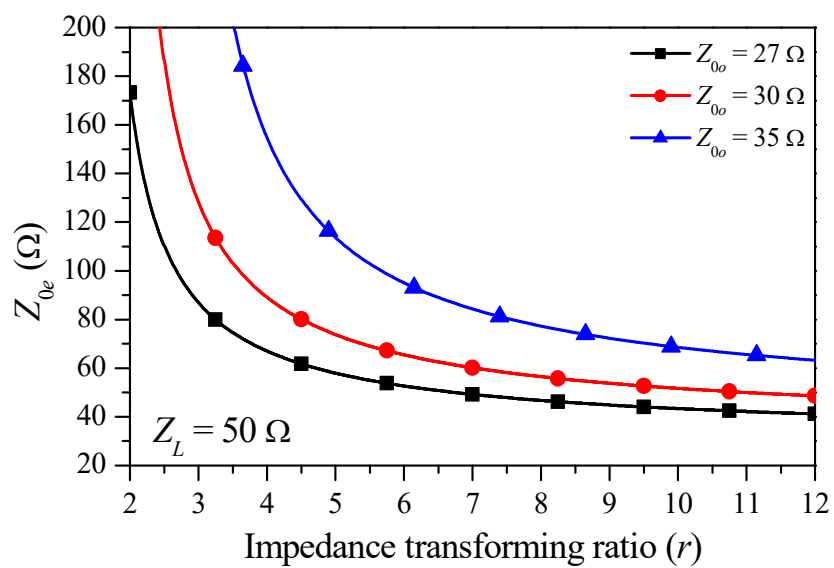

Fig. 2. Variations of $Z_{0 e}$ according to $r$ and $Z_{0}$.

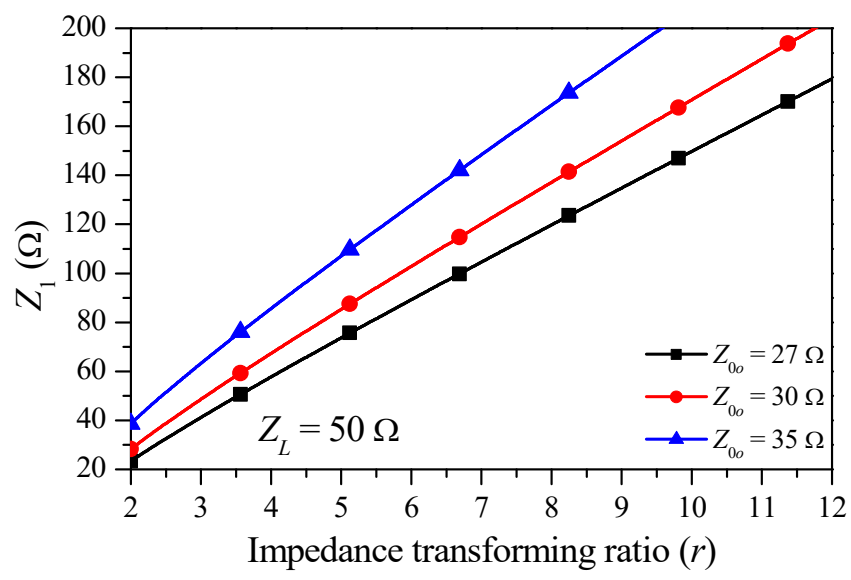

Fig. 3. Variations of $Z_{1}$ according to $r$ and $Z_{0}$.

the realization of the coupled line, whereas a high $r$ hinders the realization of a short-circuit stub TL. Therefore, a trade-off between $r$ and the characteristic impedances of $Z_{1}$ and $Z_{0 e}$ is required.

Fig. 4 shows the $S$-parameter characteristics of the proposed IT with different $r$. The stopband attenuation is improved with a high $r$; but the bandwidth of the passband becomes narrow. Moreover, two transmission poles occur in the passband.

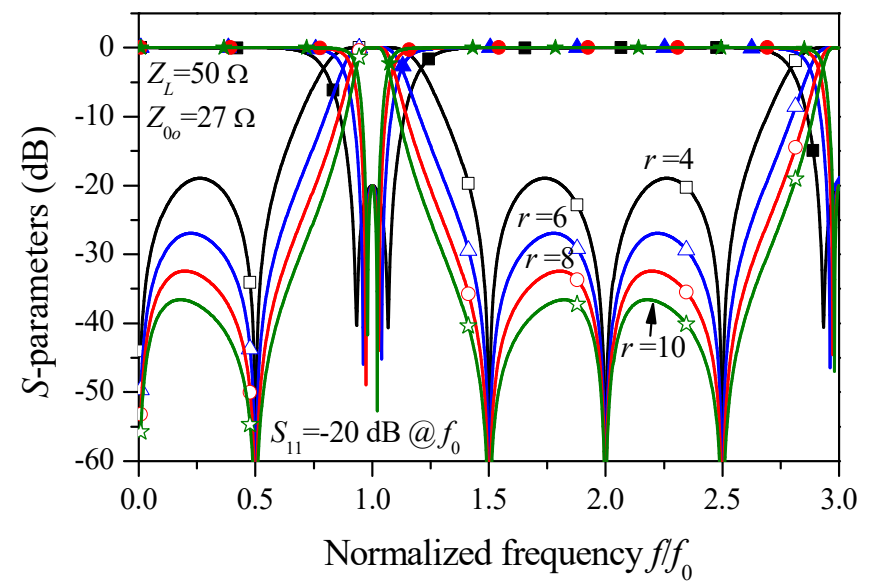

Fig. 4. $S$-parameter characteristics with different $r$.

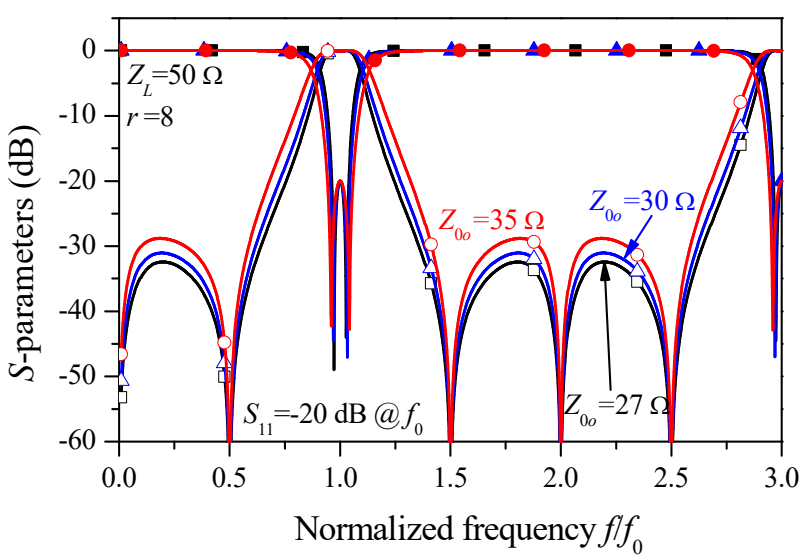

Fig. 5. $S$-parameter characteristics with different $Z_{0}$.

Table 1. Calculated variables of the proposed impedance transformer

\begin{tabular}{cccc}
\hline & \multicolumn{3}{c}{$Z_{L}=50 \Omega$ and $S_{11}=-20 \mathrm{~dB}$ at $f_{0}$} \\
\cline { 2 - 4 } & $Z_{0 o}(\Omega)$ & $Z_{0 e}(\Omega)$ & $Z_{1}(\Omega)$ \\
\hline$r=4$ & 27 & 66.99 & 57.73 \\
$r=6$ & 27 & 52.68 & 89.25 \\
$r=8$ & 27 & 46.72 & 119.78 \\
& 30 & 56.5 & 137.17 \\
& 35 & 77.3 & 168.64 \\
$r=10$ & 27 & 43.38 & 149.77 \\
\hline
\end{tabular}

Transmission zeros are produced in the stopband, and a wide stopband characteristic can be obtained. Transmission zeros at $0.5 f_{0}, 1.5 f_{0}$, and $2.5 f_{0}$ are produced by a short-circuit stub TL. Moreover, a transmission zero at $2 f_{0}$ is produced by a coupled line. The simulation is conducted by fixing $Z_{0_{0}}=27 \Omega$ and varying $r=4,6,8$, and 10. Fig. 5 illustrates the $S$-parameter characteristics of the proposed IT with different $Z_{00}$. The bandwidth of a $20 \mathrm{~dB}$ return loss is improved with a high $Z_{0}$, but the stopband attenuation is degraded. The simulation is conducted by fixing $r$ $=8$ and varying $Z_{0_{o}}=27 \Omega, 30 \Omega$, and $35 \Omega$. The calculated values of all variables of the simulation in Figs. 4 and 5 are shown in Table 1.

\section{SIMULATION AND MEASUREMENT RESULTS}

An experimental validation was conducted by designing the IT and fabricating it on an RT 5880 substrate, with $\varepsilon_{r}=2.2$ and $h=$ $0.787 \mathrm{~mm}$. The proposed IT network with a 400-50 $\Omega(r=8)$ of termination impedance, $Z_{0 \mathrm{o}}=27 \Omega$, and reflection coefficient of $20 \mathrm{~dB}$ at $f_{0}=2.6 \mathrm{GHz}$ was designed. All the calculated variables are listed in Table 1. An electromagnetic (EM) simulation was performed using the Ansoft HFSS v15.

Fig. 6 shows the EM simulation layout and photograph of the fabricated IT network. The physical dimensions of the fabricated circuit are listed in Table 2. To minimize the circuit size, a halfwavelength short-circuit stub TL is designed as a mean- 


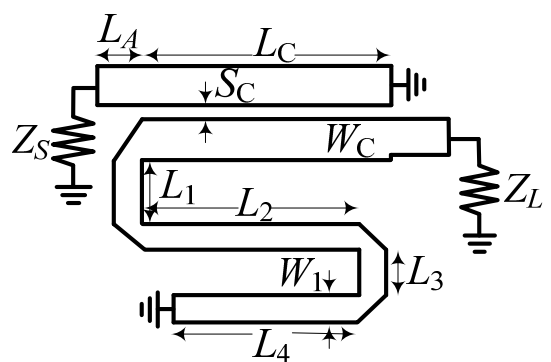

(a)

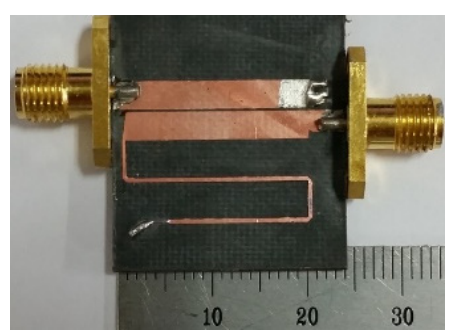

(b)

Fig. 6. The fabricated IT: (a) EM simulation layout and (b) photograph.

Table 2. Physical dimensions of the fabricated IT (unit: $\mathrm{mm}$ )

\begin{tabular}{cccc}
\hline Parameter & Value & Parameter & Value \\
\hline$W_{c}$ & 3.1 & $L_{1}=L_{3}$ & 4 \\
$W_{1}$ & 0.4 & $L_{2}$ & 19 \\
$S_{c}$ & 0.1 & $L_{4}$ & 15.7 \\
$L_{c}$ & 19.2 & $L_{\mathrm{A}}$ & 1.5 \\
\hline
\end{tabular}

der structure. The overall circuit size of the proposed IT is 24 $\mathrm{mm} \times 16 \mathrm{~mm}$.

Fig. 7 illustrates the EM simulation and measurement results. The measured results are in good agreement with the simulations. The insertion and return losses are obtained $0.6 \mathrm{~dB}$ and $22.5 \mathrm{~dB}$ at $f_{0}$, respectively. Similarly, the bandwidth of the $20 \mathrm{~dB}$ return loss is $0.19 \mathrm{GHz}$, which extends from $2.51 \mathrm{GHz}$ to $2.7 \mathrm{GHz}$. The insertion loss of the passband is better than $0.8 \mathrm{~dB}$. Moreover, one transmission zero on the lower side and three trans-

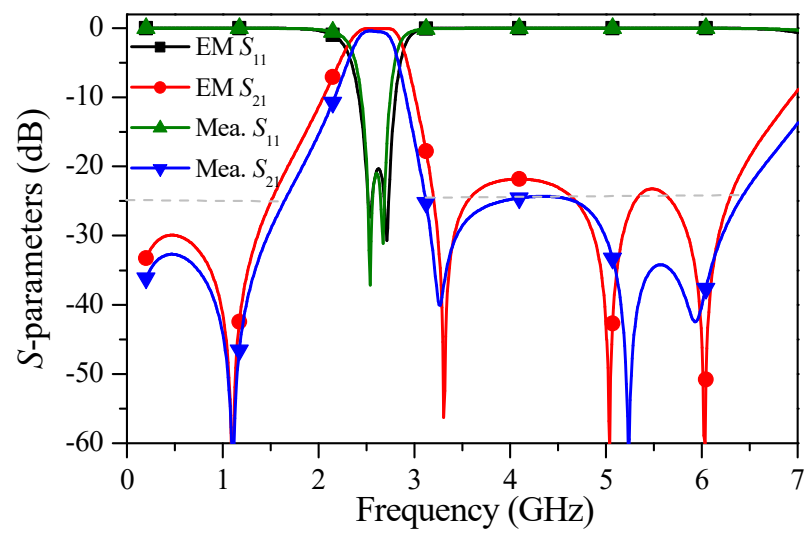

Fig. 7. EM simulation and measurement results of the proposed IT. mission zeros on the upper side of the passband are obtained, and these transmission zeros provide a wide stopband characteristic. The lower- and upper-side stopband attenuations of $25 \mathrm{~dB}$ are obtained from dc to $1.64 \mathrm{GHz}$ and from $3.12 \mathrm{GHz}$ to $6.4 \mathrm{GHz}$, respectively.

\section{CONCLUSION}

An IT with a bandpass filtering response for high termination impedance is proposed and demonstrated in this study. The designed equations are derived with a predefined return loss. To show the validity of the proposed analysis, an IT is designed, fabricated, and measured. The simulated and measured results agree well with the analysis. The proposed IT with high selectivity, multi-transmission zeros, and bandpass response can be obtained simultaneously. The proposed IT is simple to design and is expected to be applied in various applications, such as baluns, power dividers, and antenna feeding lines.

\section{REFERENCES}

[1] P. Kim, J. Jeong, G. Chaudhary, and Y. Jeong, "A design of unequal termination impedance power divider with filtering and out-of-band suppression characteristic," in Proceedings of the 45th European Microwave Conference (EuMC), Paris, France, 2015, pp. 123-126.

[2] B. J. Xiang, S. Y. Zheng, Y. M. Pan, and Y.X. Li, "Wideband circularly polarized dielectric resonator antenna with bandpass filtering and wide harmonics suppression response," IEEE Transactions on Antennas and Propagation, vol. 65, no. 4, pp. 2096-2101, 2017.

[3] B. Zhang, G. Dong, Y.Wu, C.Yu, and Y.Liu, "Filtering pushpull power amplifier based on novel impedance transformers," Electronics Letters, vol. 52, no. 17, pp. 1467-1468, 2016.

[4] G. L. Matthaei, L. Young, and E. M. T. Jones, Microwave Filters, Impedance-Matcbing Network and Coupling Structures. Norwood, MA: Artech House, 1980.

[5] R. Zhang, S. Luo, and L. Zhu, "Synthesis and design of mixed lumped and distributed low-pass filters/low-passing impedance transformers with Taylor series," IEEE Transactions on Microwave Theory and Techniques, vol. 64, no. 4, pp. 12651272, 2016.

[6] K. S. Ang, C.H.Lee, and Y.C. Leong, "A broad-band quarter wavelength impedance transformer with three reflection zeros with passband," IEEE Transactions on Microwave Theory and Techniques, vol. 52, no. 12, pp. 2640-1644, 2004.

[7] Q. S. Wu and L. Zhu, "Wideband impedance transformers on parallel-coupled and multisection microstrip lines: synthesis design and implementation," IEEE Transactions on Components, Packaging and Manufacturing Technology, vol. 6, 
no. 12, pp. 1873-1880, 2016.

[8] P. Kim, J. Park, J. Jeong, S. Jeong, G. Chaudhary, and Y.Jeong, "High selective coupled line impedance transformer with second harmonic suppression," Journal of Electromagnetic Engineering Science, vol. 16, no. 1, pp. 13-18, 2016.

[9] P. Kim, G. Chaudhary, and Y. Jeong, "Ultra-high transforming ratio coupled line impedance transformer with bandpass response," IEEE Microwave and Wireless Components Letters, vol. 25, no. 7, pp. 445-447, 2015.

[10] Q.S.Wu and L. Zhu, "Short-ended coupled-line impedance

\section{Phirun Kim (S'11-M'17)}

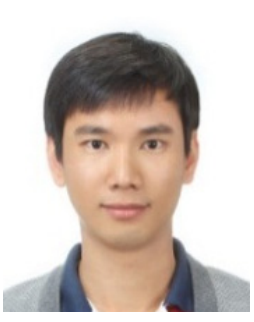

received his B.E. degree in electronics engineering from the National Polytechnic Institute of Cambodia (NPIC), Phnom Penh, Cambodia, in 2010, and his M.E. and Ph.D. degrees in electronics engineering from Chonbuk National University, Jeonju, Republic of Korea, in 2013 and 2017, respectively. He is currently a post-doctoral researcher at HOPE-IT Human Resource Development Center-BK21 PLUS, Division of Electronics Engineering, Chonbuk National University. He has authored and co-authored over 40 papers in international journals and conference proceedings. His research interests include RF filters, power dividers, impedance transformers, baluns, and high-efficiency power amplifiers. transformers with ultrahigh transforming ratio and bandpass selectivity suitable for large load impedances," IEEE Transactions on Components, Packaging and Manufacturing Technology, vol. 6, no. 5, pp. 767-774, 2016.

[11] P. Kim, G. Chaudhary, and Y.Jeong, "Enhancement impedance transforming ratios of coupled line impedance transformer with wide out-of-band suppression characteristics," Microwave and Optical Technology Letters, vol. 57, no. 7, pp. 1600-1603, 2015.

Yongchae Jeong (M'99-SM'10)

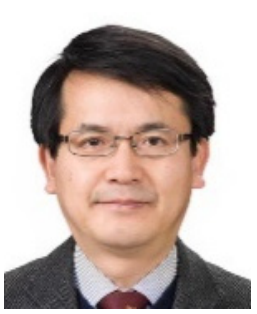

received his BSEE, MSEE, and Ph.D. degrees in electronics engineering from Sogang University, Seoul, Republic of Korea in 1989, 1991, and 1996, respectively. From 1991 to 1998, he worked as a senior engineer at Samsung Electronics. In 1998, he joined the Division of Electronics Engineering, Chonbuk National University, Jeonju, Korea. From July 2006 to December 2007, he was a visiting professor at Georgia Institute of Technology. Currently, he is a professor, member of the IT Convergence Research Center, and director of HOPE-IT Human Resource Development Center of BK21 PLUS in Chonbuk National University. He currently teaches and conducts research on microwave passive and active circuits, mobile and satellite base station RF system, design of periodic defected transmission line, negative group delay circuits and its applications, in-band full duplex radio, and RFIC design. Prof. Jeong is a senior member of IEEE and is a member of the Korea Institute of Electromagnetic Engineering and Science. He has authored and co-authored over 170 papers in international journals and conference proceeding. 